\title{
A two-score composite program for combining standard scores
}

\author{
LARRY D. EVANS \\ University of Arkansas for Medical Sciences, Little Rock, Arkansas
}

\begin{abstract}
It is often desirable for mental health practitioners to combine standard scores from different tests, raters, or times into a single composite standard score. Most often the result is a more reliable and accurate standard score. This paper describes a computer program that uses two standard scores, score reliability and correlation with a third variable, to yield a composite standard score, reliability and correlation. Trends, limitations, optimum benefits, and examples are discussed. References are provided for calculating composites based on more than two scores.
\end{abstract}

Composite scores are routinely used by test publishers to derive an overall scale score from two or more subtests. The Full Scale IQ (FSIQ) of the Wechsler Intelligence Scale for Children-Third Edition (Wechsler, 1991), the Basic Reading Skills Cluster of the Woodcock-Johnson Psychoeducational Battery-Revised (Woodcock \& Johnson, 1989), and the Adaptive Behavior Composite of the Vineland Adaptive Behavior Scales (Sparrow, Balla, \& Cicchetti, 1984) are common examples of composite scores. While often used by test publishers, composite scores are less frequently derived by mental health practitioners. This is unfortunate, as an understanding of composite scores enhances the interpretation of composite scores, such as the FSIQ, and provides a tool that may be required to synthesize psychological results.

Combining two or more same-domain scores into a single composite score offers several advantages over interpretation of individual scores. First, one conceptualization of a composite score is a score from a single test composed of the items of several individual tests (Thorndike, Cunningham, Thorndike, \& Hagen, 1991). This "longer" test generally results in a more reliable score. Second, composite scores avoid loss of information. For example, when two tests measure the same domain and yield different scores, practitioners often try to determine which score is more accurate, often by comparing scores with an overall profile, selecting the more reliable or comprehensive score, or relating subject behavior, during testing, to each score. Regardless of what single score is thought to be the more accurate, the net effect of using only one test is loss of information. Because the composite score is based on the scores of both tests, there is no sacrificing of test data. Third, composite scores offer great flexibility in test result interpretation. Weights can be assigned to scores on the basis of the purpose of the composite score.

A copy of the compiled program is available from the author upon receipt of a self-addressed, postage-paid mailer and one DOS-formatted, high-density floppy disk. Correspondence should be addressed to Larry Evans, Dennis Developmental Center, 1612 Maryland Street, Little Rock, AR 72202 (e-mail: levans@ddcchild.ach.uams.edu).
Wang and Stanley (1970) reviewed various methods to derive weights, but basic techniques may be based on reliability coefficients, test length, optimal correlation with a criterion, or subjective judgments of the importance of each test's contribution to a composite.

There are specific instances in which composite scores may be highly desirable. First, practitioners may give a second test to confirm an initial test's results, then need to combine the scores to reflect the best indication of overall performance in a particular domain. Indeed, federal and most state special education guidelines discourage reliance on a single score or procedure during decision making. The State of Arkansas, for example, requires that after an initial achievement test has been administered to a student suspected of having a learning disability, a second achievement test must be given to further assess the area of suspected disability (Arkansas Department of Education, 1993). Second, after assessing a subject, a practitioner may wish to obtain results of a recent evaluation in some other setting, either incidentally or as a blind second opinion, and to combine same-domain scores to obtain a more accurate picture of the subject's overall abilities. Third, practitioners may use screening or abbreviated test batteries, then later provide more comprehensive evaluation for failed screenings. It is often desirable to then combine the results of the screening and comprehensive scores (generally weighting the comprehensive score more heavily) so as not to lose the time and expense required for the screening. Fourth, comprehensive behavioral assessment may include subject ratings from several individuals, times, or settings. A composite score may then be needed to summarize the ratings and increase overall reliability.

\section{Statement of the Problem}

Most practitioners would benefit from being able to combine scores into a composite score. The resulting composite score would often provide a more reliable and valid summary score. Better understanding of composite scores would also aid in interpreting the composite scores provided by tests. Unfortunately, the equations needed to 
calculate composite scores are not readily available to most practitioners and are not reliably performed by hand calculations.

\section{Response to the Problem}

This paper describes a computer program designed to calculate a standard score composite based upon two standard scores. A two-score composite may be the most common composite and, as the simplest composite, provides a basis for assessing the relative benefits of multiplescore composites.

\section{COMPOSITE SCORE PROGRAM}

A program was written using Version 1.0 of Microsoft Visual Basic for MS-DOS. After an initial start-up screen of instructions, the program displays a single screen with edit fields that allow the user to enter data for two test scores (standard score, reliability, correlation with a criterion, and score weight) and the correlation between the two scores. After a push button is clicked, the calculations are performed and the composite standard score, its reliability, and its correlation with a criterion are shown at the bottom of the screen. A single, pull-down menu allows the results to be printed, entered data to be erased, or exiting the program.

The program is intentionally straightforward, with an emphasis on application. A key to successfully using the program is an understanding of the equations and output. Therefore, the following equations and examples, which are intended to help the user understand and utilize the program results, are provided.

\section{Calculating the Composite Standard Score}

The first calculation involves determining a composite score:

$$
x_{c}=b_{1} x_{1}+b_{2} x_{2},
$$

where $x_{c}$ is the composite score, $b_{1}$ and $b_{2}$ are weights, and $x_{1}$ and $x_{2}$ are the obtained standard scores. If equal weights of 1.00 are assigned to each obtained score, then Equation 1 simplifies to the sum of the obtained scores.

The composite score of Equation 1 is not a standard score in the same metric as the obtained scores, and requires that the composite's mean and standard deviation be calculated before it can be interpreted. The equation for the composite score's mean is

$$
M_{x_{c}}=b_{1} M_{x_{1}}+b_{2} M_{x_{2}},
$$

where $M_{x_{c}}$ is the composite mean, and $M_{x_{1}}$ and $M_{x_{2}}$ are means for the obtained standard scores. The composite's standard deviation is given by the equation

$$
S D_{x_{c}}=\sqrt{b_{1}^{2} S D_{x_{1}}^{2}+b_{2}^{2} S D_{x_{2}}^{2}+2} b_{1} b_{2} r_{12} S{\overline{S x_{1}} S D_{x_{2}}}
$$

where $S D_{x_{\mathrm{c}}}$ is the composite's standard deviation, $S D_{x_{1}}$ and $S D_{x_{2}}$ are the standard scores' standard deviations, and $r_{12}$ is the Pearson product-moment correlation coefficient for the standard scores. If the obtained standard scores are initially expressed as $z$ scores and equal weights of 1.00 are assigned, then Equation 3 simplifies to

$$
S D_{x_{c}}=\sqrt{2+2 r_{12}} \text {. }
$$

Equations 3 and 4 indicate that, for obtained scores with the same standard deviation, as the correlation increases toward 1.00 , the composite standard deviation approaches twice the obtained scores' standard deviation.

The final step is to convert the composite score to a standard score with the same mean and standard deviation as one or both of the obtained scores. This involves first converting the composite score to a $z$ score, then to a standard score in the same metric as one or both of the obtained scores. Using the above equations, the $z$ score for the composite score is

$$
z_{x_{c}}=\frac{x_{c}-M_{x_{c}}}{S D_{x_{c}}} .
$$

The $z$ score for the composite $\left(z_{x_{c}}\right)$ can be multiplied by the standard deviation of an obtained score and the product then be added to the same obtained score's mean in order to produce a composite standard score that is interpretable relative to the obtained scores.

One synthesis of the above equations is that, as the correlation $r_{12}$ increases toward 1.00 , the composite standard score approaches the (weighted) average of the obtained scores. As the correlation decreases, the composite standard score increasingly deviates from this average, with the direction of deviation being away from the (weighted) mean.

\section{Calculating the Composite's Reliability}

The composite standard score, as with any standard score, should not be interpreted without taking the error of measurement into account (Salvia \& Ysseldyke, 1991). The composite's reliability is therefore important for determining the standard error of measurement and the estimated true composite score. The composite's reliability may also be important to determine if the score differs significantly from another standard score (Payne \& Jones, 1957) or if the composite score is to be entered into a software analysis program such as regression software for learning disabilities (e.g., Evans, 1994). The composite's reliability also has meaning if only when compared with the reliabilities of the scores forming the composite. As will be seen, composite reliabilities that vary markedly from those of its contributing scores provide information about the relationship between those scores and their suitability in forming a composite.

The equation for the reliability of the composite standard score is given as 


$$
r_{x_{c}}=\frac{b_{1}^{2} r_{x_{1}}+b_{2}^{2} r_{x_{2}}+2 b_{1} b_{2} r_{12}}{b_{1}^{2}+b_{2}^{2}+2 b_{1} b_{2} r_{12}},
$$

where $r_{x_{c}}$ is the composite's reliability, and $r_{x_{1}}$ and $r_{x_{2}}$ are the reliabilities for the obtained standard scores. When the weights are set to 1.00 , Equation 6 becomes

$$
r_{x_{c}}=\frac{r_{x_{1}}+r_{x_{2}}+2 r_{12}}{2+2_{r_{12}}}
$$

With weights, scores, means, standard deviations, and reliabilities held constant, it is evident from Equations 4, 5 , and 7 that, as the correlation between measures decreases toward zero, the composite standard score deviates more from the mean and the reliability approaches the average reliability of the measures. As the correlation increases, the composite standard score deviates less from the mean and composite reliability increases.

\section{Calculating the Composite's Correlation With a Criterion}

In addition to increased reliability, a composite score may also provide higher correlation with a criterion score and as a result better prediction. The composite correlation coefficient is:

$$
r_{x y c}=\frac{b_{1} r_{13}+b_{2} r_{23}}{\sqrt{b_{1}^{2}+b_{2}^{2}+2 b_{1} b_{2} r_{12}}},
$$

where $r_{x y c}$ is the correlation coefficient between the composite and criterion score, $r_{13}$ is the correlation coefficient between the scores of one test and the criterion, and $r_{23}$ is the correlation between the scores of a second test and the criterion.

\section{SAMPLE CALCULATIONS OF COMPOSITE SCORES}

As part of a psychoeducational evaluation, a school psychologist administers a general achievement test and a reading test. Both tests yield several scores, including reading comprehension scores with a mean of 100 and standard deviation of 15 . Say a student received a standard score of 75 for the general achievement test's readingcomprehension subtest that was based primarily on items measuring literal comprehension of words, phrases, and short sentences. The standard score of 69 from the readingcomprehension portion of the reading test included items measuring inferential comprehension, reorganization, and critical reading for paragraphs and short passages. A composite reading comprehension score is desired because of the complementary test items and comprehensiveness of a combined assessment. Both scores are given a weight of 1.00 . The respective test manuals indicate an internal consistency reliability coefficient for the score of 75 as .90 and for 69 as .93 . Both test manuals also indicate correlations of .65 and .68 , respectively, with the intelligence test given to the student. A concurrent valid- ity study in one test manual shows a correlation between reading comprehension portions of both tests as . 78 for a nonreferred sample.

The data are entered into the composite score program. The results show a composite standard score of 70.32 with a reliability of .95 and correlation with the IQ score of .70. The achievement composite score can then be used to determine if a severe discrepancy exists between IQ and achievement.

Table 1 provides several examples of composite-score program results. The obtained scores of Table 1 have a mean of 100 , standard deviation of 15 , and identical weights. The first group of four rows contains four pairs of matching scores with high reliability and correlation for each pair. The result is a composite standard score that hardly differs from the obtained standard scores and has only a small increase in reliability. The second group of scores shows the same scores with the same high reliability but only a moderate correlation. The composite standard scores are lower than those of the first group, demonstrating the tendency for composite standard scores to move farther away from the average of the obtained scores as the correlation decreases. The composite reliability for the second group is lower than that for the first, although to two decimal places they are the same.

Table 1

\begin{tabular}{|c|c|c|c|c|}
\hline \multicolumn{3}{|c|}{ Obtained Scores } & \multicolumn{2}{|c|}{ Composite Scores } \\
\hline $\begin{array}{c}\text { Standard } \\
\text { Scores }\end{array}$ & Reliability* & Correlation & $\begin{array}{c}\text { Standard } \\
\text { Score }\end{array}$ & Reliability \\
\hline 100,100 & $.95, .95$ & .90 & 100 & .97 \\
\hline $85, \quad 85$ & $.95, .95$ & .90 & 85 & .97 \\
\hline $70, \quad 70$ & $.95, .95$ & .90 & 69 & .97 \\
\hline $55, \quad 55$ & $.95, .95$ & .90 & 54 & .97 \\
\hline 100,100 & $.95, .95$ & .75 & 100 & .97 \\
\hline $85, \quad 85$ & $.95, .95$ & .75 & 84 & .97 \\
\hline $70, \quad 70$ & $.95, .95$ & .75 & 68 & .97 \\
\hline $55, \quad 55$ & $.95, .95$ & .75 & 52 & .97 \\
\hline 100,100 & $.80, .80$ & .75 & 100 & .89 \\
\hline 85,85 & $.80, .80$ & .75 & 84 & .89 \\
\hline $70, \quad 70$ & $.80, .80$ & .75 & 68 & .89 \\
\hline $55, \quad 55$ & $.80, .80$ & .75 & 52 & .89 \\
\hline 100,100 & $.90, .90$ & .40 & 100 & .93 \\
\hline $85, \quad 85$ & $.90, .90$ & .40 & 82 & .93 \\
\hline $70, \quad 70$ & $.90, .90$ & .40 & 64 & .93 \\
\hline $55, \quad 55$ & $.90, .90$ & .40 & 46 & .93 \\
\hline 100,100 & $.70, .70$ & .40 & 100 & .79 \\
\hline $85, \quad 85$ & $.70, .70$ & .40 & 82 & .79 \\
\hline $70, \quad 70$ & $.70, .70$ & .40 & 64 & .79 \\
\hline $55, \quad 55$ & $.70, .70$ & .40 & 46 & .79 \\
\hline 100,80 & $.90, .85$ & .70 & 89 & .93 \\
\hline $85, \quad 70$ & $.85, .85$ & .60 & 75 & .91 \\
\hline $60, \quad 70$ & $.88, .92$ & .75 & 63 & .94 \\
\hline 115,125 & $.90, .90$ & .70 & 122 & .94 \\
\hline 115,90 & $.90, .85$ & .67 & 103 & .93 \\
\hline
\end{tabular}

Composite Scores and Reliabilities Based on Two Equally Weighted Standard Scores

Note-For illustrative purposes, all obtained scores have a mean of 100 and a standard deviation of 15 . *Internal consistency reliability coefficients are shown. 
The third group of obtained scores is the same as the second except for lower reliability values. Because reliability does not directly affect the composite standard score, these scores can be taken as the same as those for the second group. Of the same three groups, however, the third group's composite reliability shows a greater absolute increase over its obtained scores. This is primarily due to the fact that it had greater room for improvement than did the second group, but also to the fact that its correlations were closer to its reliabilities. That is, as the upper bound for the correlation is the lower of the reliabilities, the composite reliability increases as the correlation increases (Equation 7) and reaches its upper limit as the correlation approaches the lower of the reliability coefficients.

The fourth group of scores shown in Table 1 has high reliabilities but lower correlations than the other groups. As expected, the composite standard scores are farther away from the average of the obtained scores, and the composite's increase in reliability over the obtained scores is small, as the correlation is closer to zero than the lower reliability value. When the reliabilities decrease and the correlation remains the same (as in, e.g., the fifth group of scores), the composite standard scores predictably remain the same, but the composite reliability shows a greater relative, though smaller proportional, increase.

The first five groups of scores had identical scores of matching reliabilities, a convenience selected to allow only reliabilities or correlations to vary. The sixth group of scores shows the more common outcome of differences in obtained scores and reliabilities. Although less evident than with the first five groups, the same tendencies are found-the deviation of the composite standard score and reliability from the average of the obtained scores is directly related to the size of the correlation between scores.

Many practitioners expect that when two obtained scores are identical, the composite score will equal the obtained scores. As Table 1 shows, this is true only when the obtained scores equal the mean (or for the improbable case of two perfectly reliable and correlated scores). Otherwise, the composite score is more extreme, that is, farther from the mean, than the average of the obtained scores.

\section{DISCUSSION}

This paper describes a program used to derive a composite standard score based on two standard scores. The equations of the program are presented to enhance understanding and application. For the equations showing the general case for determining composite standard scores, reliabilities, and correlations, the reader is referred to Dunteman (1984) and Mosier (1943). Equation complexity grows considerably when more than two scores are used to derive a composite. For example, the composite's standard deviation alone includes 8 terms for a two-score composite, 18 for a three-score composite, and 36 for a four-score composite. From the discussion of Table 1, it is clear that composites based on more than two scores may not offer appreciable increases in reliability except in cases where no measures with high reliability exist and the correlation between measures is at least moderate.

In what circumstances would the composite-score program be most beneficial? From Table 1 it appears that composite scores may offer the most benefits in two primary circumstances. The first occurs when a highly reliable test does not exist to assess a particular domain but two or more moderately reliable measures of moderate correlation do exist. The resulting increase in composite reliability may overcome the absence of a highly reliable instrument. The second circumstance occurs when two or more tests complement each other and a composite is needed to obtain a comprehensive score. Such a circumstance may occur, for example, when tests assess different areas of the same domain, raters measure the same domain, or the same information is gathered at two different times.

Previous discussion has based weights upon a test feature (e.g., reliability) or a desired contribution to the composite score. Such weighting methods may not be desirable when scores are correlated. In such cases, it is possible to calculate optimal weights such as those used in multiple regression equations. Govindarajulu (1988) presents a comparison of the equations and outcomes of optimal and other weighting methods. Optimal weights provide the highest correlation between the composite standard score and a criterion. Gains in correlation, reliability, and so forth, from derived weights may offer only negligible improvement over equal weighting (Guilford, 1954), resulting in a need to match any weighting method with the composite's role in the assessment and to select weights on an a priori basis to avoid data manipulation.

Several limitations to composite scores exist. The equations and discussion presented have assumed linear, rather than curvilinear, relationships between scores. It also would be inappropriate to include a score from a spoiled or suspect test, even if its contribution was minimized with a relatively small weight. Likewise, differences in scores due to time of assessment, evaluators, normative data, or domain should be minimized unless it is the composite's function and is reflected in the correlation between scores. Composite scores are not limited to normally distributed standard scores despite the previous emphasis (some weighting methods may assume normally distributed scores). Indeed, composite score statistics are often presented in the context of standardized student scores from classroom tests (e.g., Thorndike et al., 1991).

Practical implications of composite scores are primarily related to the integration of test results, improved reliability and validity, and the resulting impact on decision making. The primary value of composite scores may lie in increasing the technology of score interpretation and providing practitioners with an additional tool to test hypotheses and obtain a clearer picture of subjects' abilities. Therefore, future research with standard score composites may best be able to investigate the outcomes of using composite scores versus individual scores. In addi- 
tion to impact on decision making, the research could include differential improvements in agreement (e.g., interrater, classification, stability, interdomain, interinterviewer) with a criterion when composite scores are used.

\section{REFERENCES}

Arkansas Department of Education (1993). Program standards and eligibility criteria for special education. Little Rock, AR: Author. DunTEMAN, G. H. (1984). Introduction to multivariate analysis. Beverly Hills, CA: Sage.

Evans, L. D. (1994). Standard score regression comparison 3.0 [Computer software]. North Little Rock, AR: WtL.

GovindARAJULU, Z. (1988). Alternative methods for combining several test scores. Educational \& Psychological Measurement, 48, 53-60.

GuILFORD, J. P. (1954). Psychometric methods. New York: McGraw-Hill. Mosier, C. I. (1943). On the reliability of a weighted composite. Psychometrika, 8, 161-168.

Payne, R. W., \& Jones, H. G. (1957). Statistics for the investigation of individual cases. Journal of Clinical Psychology, 13, 115-121.
SalviA, J., \& YsSeldyke, J. E. (1991). Assessment (Sth ed.). Boston: Houghton Mifflin.

Sparrow, S. S., Balla, D. A., \& Cicchetti, D. V. (1984). Vineland Adaptive Behavior Scales: Survey Form. Circle Pines, MN: American Guidance.

Thorndike, R. M., Cunningham, G. K., Thorndike, R. L., \& HAgen, E. P. (1991). Measurement and evaluation in psychology and education (5th ed.). New York: Macmillan.

WANG, M. W., \& Stanley, J. C. (1970). Differential weighting: A review of methods and empirical studies. Review of Educational Research, 40, 663-705.

WECHSLER, D. (1991). Wechsler Intelligence Scale for Children-Third Edition. San Antonio, TX: Psychological Corporation.

Woodcock, R. W., \& Johnson, M. B. (1989). Woodcock-Johnson Psychoeducational Battery-Revised. Allen, TX: DLM.

(Manuscript received November 20, 1995; accepted for publication January 3,1996.) 